\title{
Commentary: Cranberries and Urinary Tract Infections: How Can the Same Evidence Lead to Conflicting Advice?
}

\author{
Eunice Mah' and DeAnn J Liska ${ }^{1 *}$ \\ 'Biofortis Innovation Services, 211 E. Lake Street, Addison, IL 60101, USA
}

Article Info

\section{Article Notes}

Received: June 30, 2016

Accepted: July 19, 2016

\section{${ }^{*}$ Correspondence:}

DeAnn J Liska, Biofortis Innovation Services, 211 E. Lake

Street, Addison, IL 60101, USA

E-mail: deann.liska@mxns.com

(C) 2016 DeAnn J Liska. This article is distributed under the terms of the Creative Commons Attribution 4.0 International License.

\section{Keywords}

Cranberry

Urinary tract infection

Meta-analysis

Review

\section{ABSTRACT}

Cranberries have long been associated with the prevention of urinary tract infection (UTI); however, meta-analyses of clinical trials on the effect of cranberry for UTI have provided conflicting results. Liska and colleagues recently examined these meta-analyses to better understand the reasons behind their disparate findings and found several methodological differences. Most notably, the populations influencing conclusions varied. For example, one analysis, which concluded cranberry was not effective for UTI (RR: 0.86; 95\% $\mathrm{Cl}: 0.71,1.04)$, had the largest contribution of results from studies in patients with complicated UTI. In contrast, another review, which included many of the same studies but weighted the evidence relatively equally across populations with complicated and uncomplicated UTI, concluded cranberry was effective (RR: $0.62 ; 95 \% \mathrm{Cl}: 0.49,0.80$ ). In both reviews, a significant benefit was noted in healthy women with recurrent UTI, particularly when two or three small trials in this population were analyzed; but, inclusion of a larger study that used a lower bacterial cut-off threshold for UTI outcome definition led to significant heterogeneity and non-significant results. These findings not only impact how we design meta-analyses, but also indicate the importance in considering the patient characteristics when generalizing findings across populations. In addition, the findings suggest additional research on cranberries in healthy women with recurrent UTI may be warranted.

\section{Text}

Cranberries have historically been associated with urinary tract health; however, evidence-based reviews have resulted in conflicting conclusions on the efficacy of cranberry for urinary tract infections (UTI). A meta-analysis examines the consistency of data across studies and is considered to be the strongest level of evidence to guide clinical practice and public health decisions. Therefore, when divergent conclusions are drawn from these studies, it becomes a challenge for clinicians and policymakers to make the most appropriate or relevant recommendations.

In a recent review ${ }^{1}$, Liska and colleagues found that the two most comprehensive meta-analyses on cranberry and $\mathrm{UTI}^{2,3}$ published conflicting conclusions despite similar research questions and search strategies. Specifically, Wang and colleagues concluded “...cranberry products were associated with protective effects against UTIS (RR: 0.62; 95\% CI: 0.49, 0.80)," particularly for women with recurrent UTIs $^{3}$. In contrast, a meta-analysis by the Cochrane Collaboration published the same year reported a RR of 0.86 (95\% CI 0.71-1.04), and concluded: "...cranberry juice is less effective than previously indicated...cranberry juice cannot currently be recommended for the 
prevention of UTIs" ${ }^{2}$. Although both reviews included data in patients with pathological conditions, in the Cochrane review, these data contributed $75.6 \%$ of the total weight, whereas the data in the Wang et al. review was weighted relatively equally across populations. Interestingly, the Cochrane analysis was an update from a 2008 report $^{4}$ that had resulted in a conclusion similar to that derived by Wang and colleagues, indicating a shift in conclusions from Cochrane.

To understand the reasons behind the disparate findings, Liska and colleagues deconstructed the methodologies used in these studies and identified three main contributing factors: (1) Differences in population characteristics; (2) Differences in data selected for extraction; and, (3) Differences in the handling of heterogeneity across the studies. With respect to the differences in populations, none of the meta-analyses found a significant reduction in relative risk for UTI for cranberry compared to control in the complicated UTI subgroups. However, the relative risk in the subgroup of women with recurrent uncomplicated UTI indicated that cranberry was more effective in this population, suggesting the beneficial effect of cranberry products may be specific to uncomplicated UTI.

The possible specificity of cranberry to uncomplicated UTI may be related to its proposed mechanism of action and the different pathogenesis of complicated vs. uncomplicated UTI. In vitro and ex vivo studies have demonstrated that cranberry inhibits the adhesion of a variety of pathogens to various surfaces in a dose-dependent fashion ${ }^{5}$. Most studies have evaluated uropathogenic type I and/or P-fimbriated E. coli, while some have also showed inhibition of adherence of urinary isolates from Proteus spp. and $P$. aeruginosa, as well as $S$. aureus, $S$. typhimurium and $E$. faecalis $^{5}$. While E. coli remains the most common causative pathogen of uncomplicated UTI, accounting for $\sim 80 \%$ of infections ${ }^{6}$, it only accounts for $\sim 21 \%$ of complicated $\mathrm{UTI}^{7}$. Thus, it is reasonable to expect that the beneficial effects of cranberry to be greater for uncomplicated UTI compared to complicated UTI. Furthermore, in contrast to the (relatively) healthy bladder environment in uncomplicated UTI, complicated UTI is associated with pathogenic conditions that increase the failure rate of treatment (and possibly, prevention). These include the presence of foreign objects (e.g., indwelling catheter), functional or anatomic abnormality of the urinary tract, and immunodeficiency (the elderly, patients with diabetes, multiple sclerosis, etc.). For example, urinary catherization creates a pathogenic environment by inducing tissue edema, hyperplasia of the urothelium, and hematuria, and provides a surface which promotes bacterial-biofilm formation ${ }^{8}$. In fact, catheterassociation UTI is the most common nosocomial infection in the U.S. ${ }^{8}$. Because of the more pathogenic environment, antibiotic treatment for complicated UTI is usually more aggressive and longer ${ }^{8}$, suggesting that interventions (such as cranberry products) that are beneficial for preventing uncomplicated UTI may not be enough for complicated UTI.

The two meta-analyses ${ }^{2,3}$ also reported substantial heterogeneity, which was associated with the inclusion of one specific study that used a lower bacteriuria cutoff than commonly employed for UTI diagnosis (e.g., $10^{3}$ colony forming units (CFU)/mL vs. $10^{5} \mathrm{CFU} / \mathrm{mL}$ ). This observation highlights another limitation faced by clinical studies on cranberry and UTI: The inconsistencies in how UTI is diagnosed and, ultimately, assessed. The FDA defines complicated UTI as "a clinical syndrome characterized by pyuria and a documented microbial pathogen on culture of urine or blood, accompanied by local and systemic signs and symptoms..."9 while the working definition of uncomplicated UTI is "a clinical syndrome in women characterized by dysuria, frequency, and/or urgency in combination with pyuria and bacteriuria"10. In addition, clinical diagnosis of uncomplicated UTI generally involves the assessment of symptoms and clinical history by a health care professional. Urine culture is not required, but is recommended as a diagnostic tool if clinical symptoms persist after receiving appropriate therapy ${ }^{11}$. Furthermore, follow-up urine culture is not required to confirm success of treatment for patients whose clinical symptoms have resolved. So, it seems that in practice, assessment of clinical symptoms alone is more commonly used both for the diagnosis and evaluation of treatment success for uncomplicated UTI.

Even when uncomplicated UTI is diagnosed on the basis of both clinical symptoms and bacteriuria, there are inconsistencies in how the results are presented. Kontiokari et al. evaluated rate of first recurrence of UTI and UTI incidence density (UTI episodes/person year) during the 12 month intervention ${ }^{12}$. Stothers assessed the number of subjects experiencing $\geq 1$ UTI during the 12 month follow up $^{13}$. Barbosa-Cesnik et al. reported recurrence rate of UTI during the 6 month follow up ${ }^{14}$. The discrepancies in how success or failure of cranberry in preventing uncomplicated UTI is evaluated will continue to be a limitation for future studies unless we can agree on how to define UTI outcomes with respect to both the technical as well as the pragmatic measurements, particularly when assessing research on UTI.

Although not discussed in the review, other inconsistencies in methodologies that need to be taken into account include the form and amount of the cranberry products, as well as the definition of recurrent UTI for participant inclusion. The studies utilized different types of cranberry products (i.e. capsules ${ }^{15}$, tablets ${ }^{13}$, powder ${ }^{16}$, and juice ${ }^{12-14}$ ) provided at different amounts. Wang et al. reported that cranberry in a form of capsule or tablet was not effective in preventing UTI recurrence $(\mathrm{RR}=0.79$; 
95\% CI: 0.44-1.44) ${ }^{3}$. Unfortunately, the analysis included patients with both complicated and uncomplicated UTI and thus, it is unclear if we would observe similar findings for uncomplicated UTI alone. In addition, the studies on recurrent UTI used different definitions for the inclusion criteria. For example, some studies included women with active confirmed UTI, treated them with antibiotics, and then began the intervention, while others used medical history documentation of at least one confirmed UTI in the previous year. These differences are reflective of the variety of definitions used in different countries and among clinicians, and demonstrate the difficulty in marrying the pragmatic with the experimental approaches.

To our knowledge, the review by Liska and colleagues is the first assessment of evidence-based reviews on cranberries and the prevention of UTI with evaluation of methodological discrepancies between the high profile meta-analyses. Findings from the review suggest that conclusions on cranberry and UTI should consider differences in the various populations studied when interpreting results from meta-analyses. The population with the most to gain from continued research into cranberry and UTI is women with history of uncomplicated UTI. This population not only has a high incidence rate of UTI recurrence, but is also met with limited alternative choices other than antibiotics, which increase concern for developing multi-drug resistant uropathogens. Furthermore, several authors ${ }^{5,17,18}$ have noted that the effect of cranberry products in women with recurrent uncomplicated UTI demonstrate the most promising results. Unfortunately, there is no comprehensive riskbenefit analysis on the use of prophylactic antibiotics compared to other alternatives on UTI, although one study did assess the potential cost effectiveness of cranberry as a prophylaxis $^{13}$. This is an important gap in knowledge that needs to be addressed to better understand the importance of investigating alternatives, such as cranberry, for UTI prevention. Many factors should be considered in weighing an approach such as cranberry to use of prophylactic antibiotic therapies for recurrent uncomplicated UTIs. Future research should take into account the limitations highlighted by Liska and colleagues if we are to ever resolve the controversy surrounding the use of cranberry products as a prophylaxis against UTI.

\section{Competing Interests}

The authors declare that they have no competing interests.

\section{References}

1. DSM-5 The American Psychiatric Association's Diagnostic and Statistical Manual, Fifth Edition (DSM-5) 2013.

2. Smith M, Flodman P, Gargus JJ, et al. Mitochondrial and ion channel gene alterations in autism. Biochim Biophys Acta. 2012 0ct; 1817(10): 1796-802

3. Moreno-De-Luca D, Sanders SJ, Willsey AJ, et al. Using large clinical data sets to infer pathogenicity for rare copy number variants in autism cohorts. Mol Psychiatry. 2013 Oct; 18(10): 1090-5.

4. Pinto D, Delaby E, Merico D, et al. Convergence of genes and cellular pathways dysregulated in autism spectrum disorders. Am J Hum Genet. 2014 May 1; 94(5): 677-94.

5. De Rubeis S, He X, Goldberg AP, et al. Synaptic, transcriptional and chromatin genes disrupted in autism. Nature. 2014 Nov 13; 515(7526): 209-15.

6. Chen JA, Peñagarikano O, Belgard TG, et al. The emerging picture of autism spectrum disorder: genetics and pathology. Annu Rev Pathol. Jan 2015; 10: 111-44.

7. Sanders SJ. First glimpses of the neurobiology of autism spectrum disorder. Curr Opin Genet Dev. 2015 Aug; 33: 80-92.

8. Bourgeron T. Current knowledge on the genetics of autism and propositions for future research. C R Biol. 2016 Jun 8. pii: S16310691(16)30041-5

9. Mahfouz A, Ziats MN, Rennert OM, et al. Shared Pathways Among Autism Candidate Genes Determined by Co-expression Network Analysis of the Developing Human Brain Transcriptome J Mol Neurosci. 2015 Dec; 57(4): 580-94.

10.Sandin S, Lichtenstein P, Kuja-Halkola $\mathrm{R}$, et al. The familial risk of autism. JAMA. 2014 May 7; 311(17): 1770-7.

11.Gaugler T, Klei L, Sanders SJ, et al. Most genetic risk for autism resides with common variation. Nat Genet. 2014 Aug; 46(8): 881-5.

12. Robinson EB, St Pourcain B, Anttila V, et al. Genetic risk for autism spectrum disorders and neuropsychiatric variation in the general population. Nat Genet. 2016 May; 48(5): 552-5. doi: 10.1038/ng.3529. PMID:26998691.

13. Robinson EB, Neale BM, Hyman SE. Genetic research in autism spectrum disorders. Curr Opin Pediatr. 2015 Dec; 27(6): 685-91. doi: 10.1097/MOP.0000000000000278. PMID:26371945. 